IOS Press

\title{
Blueberry and cranberry fruit composition during development ${ }^{1}$
}

\author{
Charles F. Forney*, Wilhelmina Kalt, Michael A. Jordan, Melinda R. Vinqvist-Tymchuk \\ and Sherry A.E. Fillmore \\ Atlantic Food and Horticulture Research Centre, Agriculture and Agri-Food Canada, Kentville, NS, Canada
}

Received 14 December 2011; accepted 22 March 2012

\begin{abstract}
Compositional changes that occur during fruit development affect both the organoleptic and nutritional quality of small fruit. Compositional changes in blueberry (Vaccinium corymbosum L) and cranberry (Vaccinium macrocarpon Aiton) fruit were determined at 3 maturities (white, turning and fully colored) during 2 seasons by analyzing sugar, acid, total phenolic, and total anthocyanin composition, ORAC antioxidant capacity, and fruit firmness. In blueberry fruit, the primary sugars were glucose and fructose, which increased as fruit ripened. Citric acid comprised 77 to $87 \%$ of the organic acids in blueberry fruit. In addition, quinic and malic acids comprised 4 to $11 \%$ of total acids and small amounts of succinic, tartaric, and shikimic acids were present. Total acids declined $68 \%$ during fruit ripening. Total phenolics were greatest in white fruit and anthocyanins were greatest in blue fruit. Antioxidant capacity declined as fruit ripened from white to turning. Fruit firmness decreased about $80 \%$ as fruit ripened. In cranberry fruit, sugar concentration increased slightly as fruit ripened with glucose comprising about $80 \%$ of the total sugars. Acid content decreased $22 \%$ during ripening primarily due to a decline in citric acid. Quinic and malic acids increased slightly during ripening. Total anthocyanins increased as color developed, while total phenolics and antioxidant capacity remained relatively constant. In contrast to blueberries, red cranberry fruit were firmer than white or turning fruit.
\end{abstract}

Keywords: Ripeness, sugars, acids, phenolics, anthocyanins, antioxidant capacity, vaccinium

\section{Introduction}

The maturation and ripening of both blueberry (Vaccinium corymbosum L.) and cranberry (Vaccinium macrocarpon Ait.) fruit affect the fruit's organoleptic properties as well as its phytonutrient composition [1,2]. To optimize fruit quality and nutritional value, it is important to understand the influence of fruit maturity on these components. Ripening can cause dramatic changes in fruit composition, but factors that control ripening in these fruit are poorly understood. In blueberry fruit, once ripening is initiated, fruit color changes rapidly on the plant, going from 50\% pink to fully blue in 2 to 3 days [3]. This is accompanied by substantial increases in anthocyanin pigment concentration as fruit change from white to dark blue in blueberries [2] or white to red in cranberries [4, 5]. Associated with color development are changes in soluble solid and acid content of the fruit [3,6], however, changes in specific sugars and acids during ripening have not been characterized. Compared to many fruits both blueberries and cranberries have abundant quantities of phenolics and high antioxidant capacity, which may contribute to their health benefits [7,8]. The objective of this study was to characterize and compare compositional changes that occur during fruit ripening of two Vaccinium species in order to improve our understanding of developmental changes that may aid in optimizing fruit quality and healthful properties.

\footnotetext{
${ }^{1}$ Paper presented at 28th International Horticulture Congress, IHC 2010, Lisbon, Portugal.

*Corresponding author: Charles F. Forney, Atlantic Food and Horticulture Research Centre, Agriculture and Agri-Food Canada, 32 Main Street, Kentville, Nova Scotia B4N 1J5, Canada. Tel.: +1 902679 5714; Fax: +1 902679 2311; E-mail: charles.forney@agr.gc.ca.
} 


\section{Material and methods}

\subsection{Fruit}

\subsubsection{Blueberry}

In the 2004 season, 'Bluecrop' blueberry fruit were sampled from 4 plots of 2 bushes selected randomly within a single commercial field located in Medford, Nova Scotia, Canada. Fruit were harvested at 3 maturity stages: white, turning (some white, pink, and blue present), and blue. Fruit were harvested from a fruit cluster only once, and approximately 30 fruit of each maturity were harvested from each plot. White fruit were harvested on July 20 , turning fruit were harvested on August 12, and blue fruit were harvested on August 17.

In the 2006 season, both 'Bluecrop' and 'Reka' highbush blueberry fruit were harvested. As in 2004, fruit were harvested from 4 plots of 2 bushes selected randomly within a single commercial field located in Medford, Nova Scotia, Canada for each cultivar. Thirty-fruit samples of each of the 3 maturities were harvested on August 14 from each plot.

\subsubsection{Cranberry}

In the 2004 season, 'Stevens' cranberry fruit were harvested from 4 plots selected randomly within a single commercial field located in Aylesford, Nova Scotia, Canada. White fruit were harvested on September 17, turning (some white and red) fruit were harvested on October 18, and red fruit were harvested on November 22. At each harvest date, 30 fruit were harvested from the top and bottom of the upright and from each of the 4 plots in the field.

In the 2006 season, 'Stevens' cranberry fruit of all 3 maturities were harvested on October 13 as described for 2004. A total of 30 white, turning, and red fruit were harvested from each plot. Fruit position on the upright was not noted since position had no significant effect on fruit composition in 2004 (results not shown).

\subsection{Firmness}

Following each harvest, fruit were assessed for firmness and then held at $-80^{\circ} \mathrm{C}$ until chemical analysis was conducted. Fruit firmness was measured using a FirmTech1 firmness testing instrument (BioWorks, Stillwater, Oklahoma, USA). Twentyfive fruit from each sample were compressed by a $15 \mathrm{~mm}$ diameter load plate at a rate of $10 \mathrm{~mm} / \mathrm{s}$ to a 2 newton $(\mathrm{N})$ threshold. The average firmness of each 25 fruit sample was recorded in $\mathrm{N} / \mathrm{mm}$ of deformation.

\subsection{Sugar, organic acid, and phenolic extraction}

Frozen fruit (15 g) were chopped using a Magic Bullet food processor (Homeland Housewares, Sherman Oaks, California, USA). A weighed amount of chopped frozen fruit was ground in a Virtis Homogenizer (The Virtis Co., Gardiner, New York, USA) for 2 min in 3 volumes of a solvent mixture containing methanol, acetone, water, formic acid $(40: 40: 20: 0.1)$. Extracts were vacuum filtered through a $9 \mathrm{~cm}$ glass fibre filter (Fisher Scientific, Nepean, Ontario, Canada), brought to volume with extraction solvent, and stored at $-80^{\circ} \mathrm{C}$ prior to sugar, acid, total phenolic and anthocyanin analysis.

A $5 \mathrm{~mL}$ sample of the extract was dried using a Savant SpeedVac Concentrator with a refrigerated condensation trap (Fisher Scientific Inc., Nepean, Ontario, Canada) and then dissolved into $5 \mathrm{~mL}$ of water, which was used directly for oxygen radical absorbance capacity assay (ORAC). A $2 \mathrm{~mL}$ sample of this water extract was loaded onto a Bond Elut C-18 $500 \mathrm{mg}$ extraction column (Varian, Lake Forest, California, USA) that was preconditioned with $5 \mathrm{~mL}$ methanol followed by $5 \mathrm{~mL}$ of water. Sugars and acids were eluted from the column with water and a $1.4 \mathrm{~mL}$ sample was collected for HPLC analysis. Phenolics and anthocyanins were then eluted from the column with methanol.

\subsection{Sugar analysis}

Sugars were analyzed using a Beckman System Gold HPLC (Beckman Instruments, Inc., Berkeley, California, USA) equipped with an Aminex HPX-87C $(300 \times 7.8 \mathrm{~mm}, 9 \mu)$ column and a Carbo-C guard cartridge $(30 \times 4.6 \mathrm{~mm})$ (Biorad Laboratories, California, USA). The column was held at $85^{\circ} \mathrm{C}$ and used a $0.6 \mathrm{~mL} / \mathrm{min}$ flow of water as the 
mobile phase. Sugars were detected using a Waters 2414 Refractive Index Detector (Waters Corporation, Milford, Massachusetts, USA) and quantified using external standards of sucrose, glucose, and fructose.

\subsection{Organic acid analysis}

Organic acids were quantified using a Beckman System Gold HPLC equipped with a Synergi Hydro-RP column $(250 \times 4.6 \mathrm{~mm}, 4$ micron) and a SecurityGuard C-18 guard cartridge (both from Phenomenex, Torrence, California, USA). The column was held at $30^{\circ} \mathrm{C}$ and acids were eluted isocratically using a $0.5 \mathrm{~mL} / \mathrm{min}$ flow of $0.01 \mathrm{M} \mathrm{H}_{2} \mathrm{SO}_{4}$ (pH 2.5). Acids were detected by absorbance at $215 \mathrm{~nm}$ using a Beckman System Gold 168 Photo Diode Array Detector and were quantified using the external standards tartaric acid, quinic acid, malic acid, shikimic acid, citric acid, and succinic acid.

\subsection{Total phenolics and anthocyanins}

Total phenolic concentration of extracts was measured spectrophotometrically at $700 \mathrm{~nm}$ using Folin-Ciocalteau reagent and gallic acid as a standard [9]. Total anthocyanin concentration of the extracts was determined by the spectrophotometric method of Giusti and Wrolstad [10] using pure cyanidin-3-glucoside (Extrasynthese, Genay, France) as a standard.

\subsection{Oxygen radical absorbance capacity}

Hydrophilic antioxidant capacity was measured using an ORAC assay, based on the method of Huang et al. [11], analyzed on a Fluoroskan Ascent FL fluorescence 96 well microplate reader (Thermo Scientific, Hudson, New Hampshire, USA). The antioxidant standard used was 6-hydroxy-2,5,7,8-tetramethyl-2-carboxylic acid (Trolox, a water soluble analogue of vitamin E) and results expressed as mmol trolox equivalents (TE) per gram fresh weight $(\mathrm{FW})$.

\subsection{Statistical analysis}

Data were analyzed using the analysis of variance (ANOVA) directive and standard error (SEM) option of GenStat ${ }^{\circledR}$ [12]. The effect of fruit position on cranberry fruit composition variates in the 2004 fruit was not significant $(P>0.05)$ and therefore this data was averaged. Similarly, there was no significant interaction between blueberry cultivars and fruit maturity in the 2006 harvest, and therefore cultivar values were averaged to show main effects of maturity.

\section{Results and discussion}

\subsection{Blueberries}

\subsubsection{Sugars}

Glucose and fructose were the predominant sugars in blueberry fruit and were found in equal quantities (Fig. 1). Trace amounts of sucrose were detected. Both glucose and fructose increased as fruit ripened $(P<0.001)$, which was also reported by Famiani et al. [13]. Total sugars in blue fruit averaged 1.5 and 3.5-fold greater than in turning and white fruit, respectively. Concentrations of glucose and fructose were similar to data presented here in ripe 'Blueray' [13], 'Coville' and 'Berkley' [14] blueberry fruit. The low concentration of sucrose in blueberry fruit may be due to invertase activity [14].

\subsubsection{Acids}

The primary organic acid found in blueberry fruit was citric acid comprising 77 to $87 \%$ of the total acids depending on maturity (Fig. 2). Substantial concentrations of malic and quinic acid were also present, each accounting for 4 to $11 \%$ of total acids. In addition, small amounts of succinic, tartaric and shikimic acids were present. Other studies 


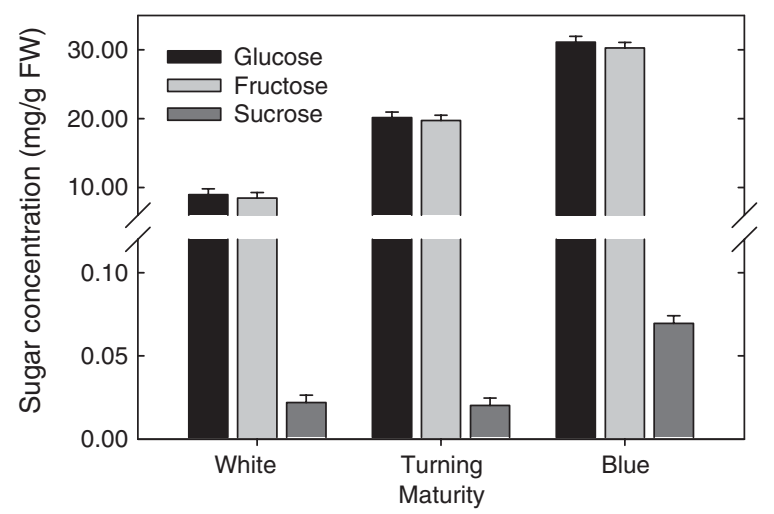

Fig. 1. The average concentration of sugars in 'Bluecrop' (2004 and 2006 seasons) and 'Reka' (2006 season) blueberry fruit harvested at 3 maturities. Values are the averages of fruit from 4 locations within each season. Error bars represent the standard error of the means $(n=12)$ for each variate.

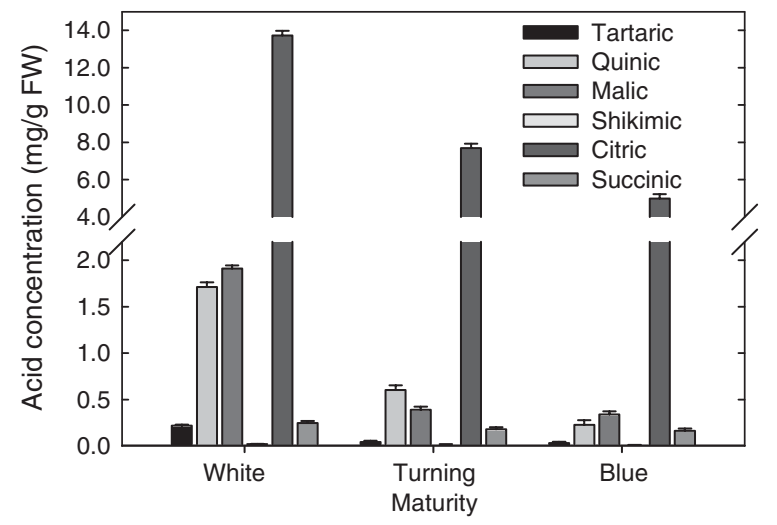

Fig. 2. The average concentration of acids in 'Bluecrop' (2004 and 2006 seasons) and 'Reka' (2006 season) blueberry fruit harvested at 3 maturities. Values are the averages of fruit from 4 locations within each season. Error bars represent the standard error of the means $(n=12)$ for each variate.

have reported citric, malic and quinic acids present in highbush blueberries $[15,16]$. All acids declined significantly as fruit ripened $(P<0.001)$. As fruit ripened from white to blue, concentrations of citric, malic, quinic, tartaric, shikimic, and succinic acids decreased 64, 82, 87, 85, 60, and 34\%, respectively. A decline in citric acid was reported in ripening 'Blueray' and 'Wolcott' blueberry fruit [13, 15]. Famiani et al. [13] also reported a decline in malic acid during blueberry fruit ripening. In other studies, fruit titratable acidity was reported to decline as fruit ripened $[6,13$, 15].

\subsubsection{Phenolics and anthocyanins}

Total phenolic concentration was highest in white fruit and decreased about $60 \%$ in turning fruit (Fig. 3). Since the white fruit in the 2004 season were harvested earlier and therefore less mature than the white fruit harvested in 2006, this difference in total phenolic concentration was greatest in white fruit from the 2004 season (data not shown). Total phenolics in blue fruit were 30\% greater than turning fruit (Fig. 3). Others have also reported the highest total phenolics in immature fruit compared to other fruit maturities [2, 6]. In the 2006 season, total phenolics in 'Bluecrop' fruit were $32 \%$ greater than in 'Reka' fruit but effects of maturity on phenolic content was similar (data not shown). Castrejón et al. [6] reported a higher concentration of total phenolics in later harvested blue fruit in 2 of 4 cultivars. Anthocyanins increased as fruit ripened, reflecting color development, and may have contributed to the rise in total 


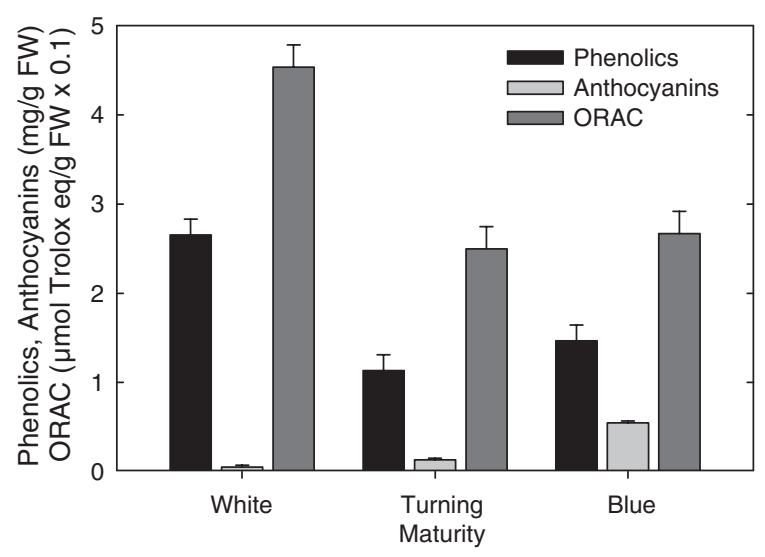

Fig. 3. The average concentration of phenolics (mg gallic acid equivalents/g FW) and anthocyanins (mg cyanidin-3-glucoside equivalents/g FW) and the antioxidant capacity (ORAC, $\mu \mathrm{mol}$ trolox equivalents/g FW) in 'Bluecrop' (2004 and 2006 seasons) and 'Reka' (2006 season) blueberry fruit harvested at 3 maturities. Values are the averages of fruit from 4 locations within each season. Error bars represent the standard error of the means $(n=12)$ for each variate.

phenolics in the blue fruit (Fig. 3). Ripe fruit had an anthocyanin concentration ranging from 0.27 to $0.83 \mathrm{mg} / \mathrm{g} \mathrm{FW}$, averaging $0.55 \mathrm{mg} / \mathrm{g} \mathrm{FW}$.

\subsubsection{Antioxidant capacity}

The ORAC values of fruit declined as fruit matured and began to turn blue (Fig. 3). The highest ORAC value was found in the least mature 2004 white fruit, which also had the highest total phenolic content. Total phenolic content has been reported to have a strong positive correlation with antioxidant capacity [7]. The ORAC values of blue fruit did not differ significantly from that of turning fruit.

\subsubsection{Firmness}

Blueberry fruit firmness decreased as fruit matured (Fig. 4). The immature white fruit were firmest averaging 4.4 and 5.9-fold firmer than the turning and blue fruit, respectively. Firmness of turning fruit was only slightly greater than that of the fully ripe blue fruit. Forney [3] reported the firmness of blue 'Coville' fruit did not change during 6 days of ripening on the bush. Therefore, it appears that most of blueberry fruit softening occurs during the white and turning stages of fruit maturation with little occurring once fruit is fully blue. This is in contrast to many climacteric fruit that soften substantially during and following color development [17].

\subsection{Cranberries}

\subsubsection{Sugars}

Unlike blueberries, glucose was the predominant sugar in cranberry fruit comprising 74 to $82 \%$ of the total sugars (Fig. 5). Fructose (16 to $24 \%$ ) and sucrose $(<0.4 \%)$ were the other sugars present. Similarly, Hong and Wrolstad [18] reported that glucose concentrations averaged about 4-times greater than that of fructose in juice extracted from red cranberry fruit representing a number of cultivars, growing locations, and seasons. Sugar concentrations increased during ripening but not to the degree seen in blueberry. Total sugars increased only 1.4-fold as fruit ripened from white to red. Glucose concentration increased 1.3-fold, but was not significant, while fructose concentration increased 2.1-fold $(P<0.001)$. Soluble solids, which reflect sugar content, also were higher in red 'Stevens' fruit $(9.2 \%)$ than white fruit (7.2\%) [19]. Total sugar content of red cranberry fruit was only about $40 \%$ of that of ripe blueberry fruit. 


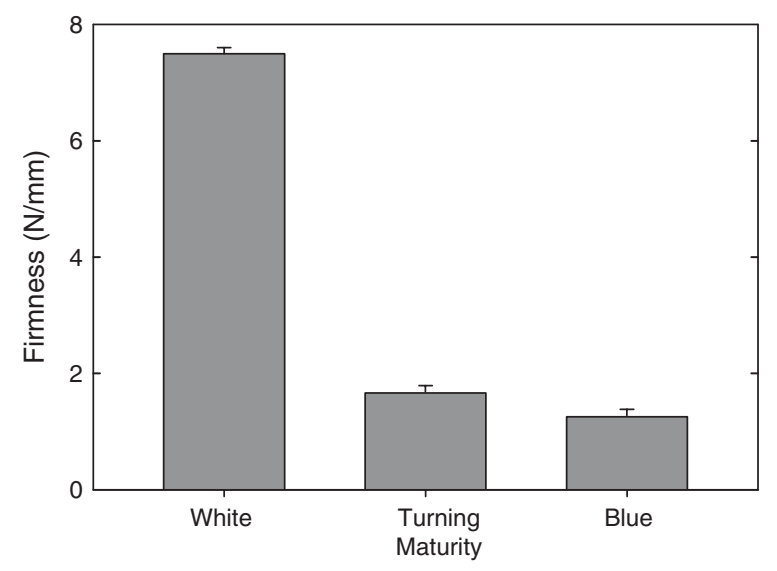

Fig. 4. The average firmness of 'Bluecrop' (2004 and 2006 seasons) and 'Reka' (2006 season) blueberry fruit harvested at 3 maturities. Values are the averages of fruit from 4 locations within each season. Error bars represent the standard error of the means $(n=12)$ for each variate.

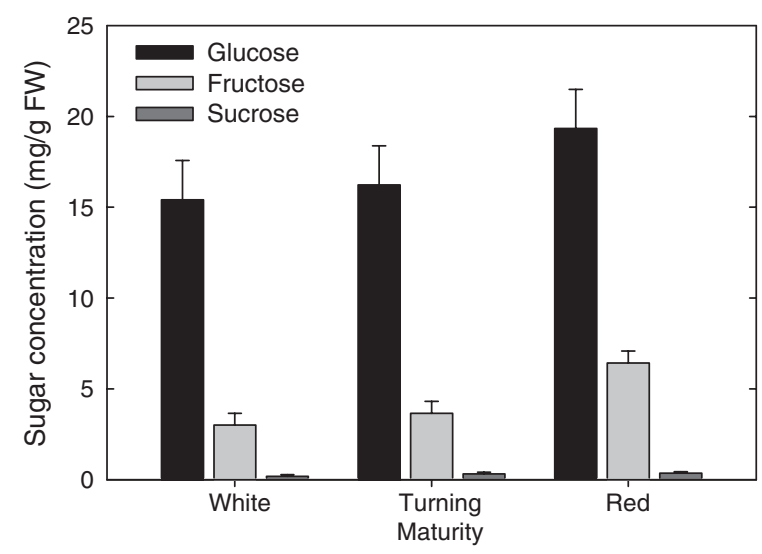

Fig. 5. The average concentration of sugars in 'Stevens' cranberry fruit harvested at 3 maturities. Values are the averages of fruit from 4 locations and 2 seasons. In the 2004 season, fruit were harvested from 2 locations on the plant. Error bars represent the standard error of the means $(n=12)$ for each variate.

\subsubsection{Acids}

The predominant organic acids in cranberry fruit were quinic, malic and citric with smaller amounts of succinic, shikimic, and tartaric present (Fig. 6). Hong and Wrolstad [18] reported that quinic, malic, citric and shikimic acids comprised an average of $39,27,32$, and $1.2 \%$ of the total acids, respectively, in juice extracted from red cranberry fruit, which was similar to the distribution we observed. During ripening, citric acid concentration decreased $63 \%$ as fruit went from white to red $(P<0.05)$. However, total acid only decreased $22 \%$, and quinic and malic acids increased 15 and $30 \%$, respectively although these changes were not significant. This retention of acidity is in contrast to blueberry fruit, which had a $68 \%$ reduction in total acids. Ripe cranberry fruit had a $75 \%$ greater total acid content than blueberry fruit helping to explain its tart taste.

\subsubsection{Phenolics}

Unlike blueberries, total phenolics only changed slightly as fruit matured from white to turning (Fig. 7). Total phenolics in red fruit were $22 \%$ greater than that in white fruit, which was associated with anthocyanin formation. Vvedenskaya and Vorsa [4] reported that the major phenolics in cranberry, flavonols and proanthocyanidins remained 


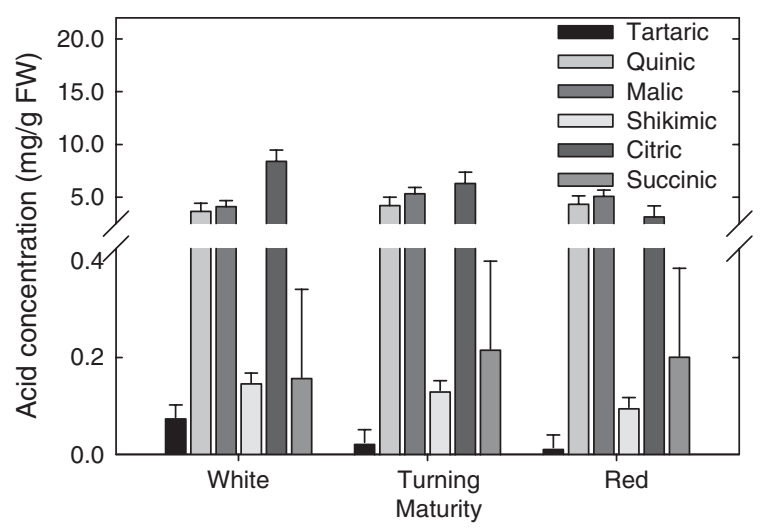

Fig. 6. The average concentration of acids in 'Stevens' cranberry fruit harvested at 3 maturities. Values are the averages of fruit from 4 locations and 2 seasons. In the 2004 season, fruit were harvested from 2 locations on the plant. Error bars represent the standard error of the means $(n=12)$ for each variate.

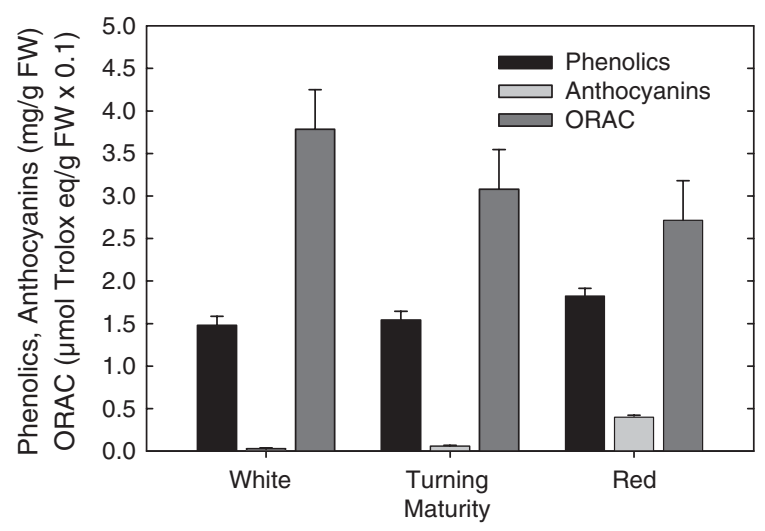

Fig. 7. The average concentration of phenolics (mg gallic acid equivalents/g FW) and anthocyanins (mg cyanidin-3-glucoside equivalents/g FW) and the antioxidant capacity (ORAC, $\mu$ mol trolox equivalents/g FW) in 'Stevens' cranberry fruit harvested at 3 maturities. Values are the averages of fruit from 4 locations and 2 seasons. In the 2004 season, fruit were harvested from 2 locations on the plant. Error bars represent the standard error of the means $(n=12)$ for each variate.

fairly constant during fruit ripening, while anthocyanins increased, which may explain the overall increase in total phenolics. While the phenolic concentration of ripe cranberries and blueberries was similar (Figs. 3 and 7), the greater astringency of cranberries may be explained by a higher concentration of proanthocyanidins [4]. The different proportion and amount of acids may also contribute to the astringent taste.

\subsubsection{Antioxidant capacity}

The ORAC of cranberry fruit declined slightly as fruit ripened (Fig. 7). This decline was in spite of the increase in total phenolics suggesting that the change in phenolic composition or changes in non-phenolic antioxidant were responsible for this change in ORAC capacity. Overall, the ORAC averaged 40 and $23 \mu \mathrm{mol}$ Trolox eq/g FW in the 2004 and 2006 seasons, respectively (data not shown).

\subsubsection{Firmness}

Unlike blueberries and most other fruit, cranberry fruit firmness increased as fruit matured and became red (Fig. 8). Firmness of red fruit was $24 \%$ greater than that of white fruit, while that of turning fruit was intermediate. It was 


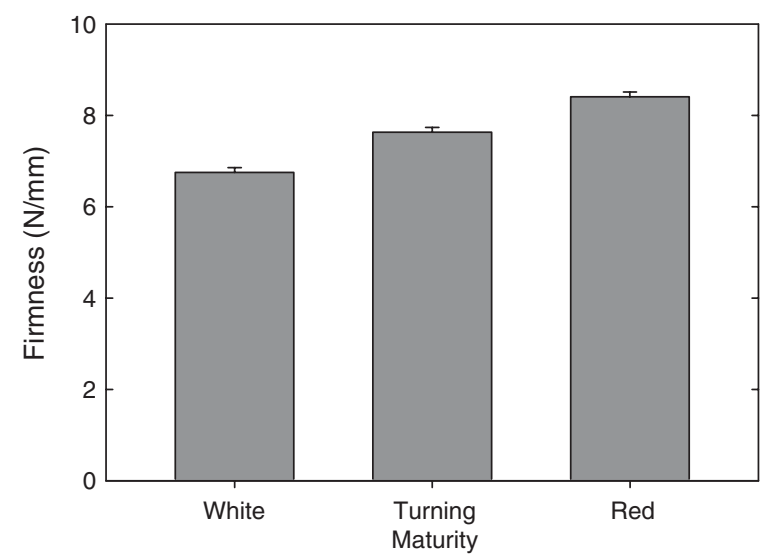

Fig. 8. The average firmness of 'Stevens' cranberry fruit harvested at 3 maturities. Values are the averages of fruit from 4 locations and 2 locations on the plant from the 2004 season. Error bars represent the standard error of the means $(n=8)$ for each variate.

previously reported that red fruit has greater resistance to puncture and a thicker cuticle than less mature fruit [19], which could contribute to this increased firmness.

\section{Conclusions}

The composition of blueberry and cranberry fruit changed substantially during ripening. Blueberry fruit had greater changes in sugar and acid composition and firmness than cranberry fruit resulting in a softer sweeter fruit at full ripeness. Since glucose is perceived as less sweet than fructose, the high glucose, low fructose content, low total sugar concentration, and high acidity in cranberry fruit appear responsible for its perceived lack of sweetness. Of all the acids measured, citric acid loss was primarily responsible for the loss of fruit acidity during ripening in both blueberry and cranberry fruit. Unlike most fruit, cranberries did not soften during ripening but rather showed a slight increase in firmness as fruit turned red.

The high phenolic content and antioxidant capacity of both cranberry and blueberry fruit, compared to other fruit [20] have been related to a variety of health benefits when these fruit are included in the diet [7, 8]. Changes in total phenolics during the ripening of blueberry and cranberry was not large, however changes in specific phenolic compounds could have greater significance on the nutritional value of these fruit. For example anthocyanin-related nutritional value may be significantly affected by ripeness stage of the berries. As other specific compounds are identified to be responsible for health benefits, their response to fruit ripening will need to be evaluated.

\section{Acknowledgments}

The authors thank Nova Agri Inc., Centreville, NS and Bezanson and Chase Cranberry Company Ltd., Aylesford, NS for supplying the blueberries and cranberries, respectively and Kathleen Munro Pennell for providing technical support.

\section{References}

[1] Forney CF. Horticultural and other factors affecting aroma volatile composition of small fruit. HortTechnology. 2001; 11(4): 529-38.

[2] Kalt W, Lawand C, Ryan DAJ, McDonald JE, Donner H, Forney CF. Oxygen radical absorbing capacity, anthocyanin and phenolic content of highbush blueberries (itVaccinium corymbosum L.) during ripening and storage. J Amer Soc Hort Sci. 2003; 128(6): 917-23.

[3] Forney CF. Postharvest issues in blueberry and cranberry and methods to improve market-life. Acta Hort. 2009; 810: 785-98. 
[4] Vvedenskaya IO, Vorsa N. Flavonoid composition over fruit development and maturation in American cranberry, Vaccinium macrocarpon Ait. Plant Science. 2004; 167(5): 1043-54.

[5] Forney CF, Kalt W, Abrams SR, Owens SJ. Effects of postharvest light and ABA treatments on the composition of late-harvested white cranberry fruit. Acta Hort. 2009; 810: 799-806.

[6] Castrejón ADR, Eichholz I, Rohn S, Kroh LW, Huyskens-Keil S. Phenolic profile and antioxidant activity of highbush blueberry (Vaccinium corymbosum L.) during fruit maturation and ripening. Food Chem. 2008; 109(3): 564-72.

[7] Kalt W, Howell AB, MacKinnon SL, Goldman IL. Selected bioactivities of Vaccinium berries and other fruit crops in relation to their phenolic contents. J Sci Food Agric. 2007; 87(12): 2279-85.

[8] Forney CF, Kalt W. Blueberry and cranberry. In: Terry LA, editor. Health-promoting Properties of Fruit \& Vegetables. Wallingford: CABI; 2011. p. 51-73.

[9] Singleton VL, Rossi JA. Colorimetry of total phenolics with phosphomolybdic-phosphotungstic acid reagents. American Journal of Enology and Viticulture. 1965; 16: 144-58.

[10] Giusti MM, Wrolstad RE. Unit F1.2.1-13. In: Wrolstad RE, editor. Current Protocols in Food Analytical Chemistry. New York: Wiley; 2001.

[11] Huang D, Ou B, Hampsch-Woodill M, Flanagan JA, Prior RL. High-throughput assay of oxygen radical absorbance capacity (ORAC) using a multichannel liquid handling system coupled with a microplate fluorescence reader in 96-well format. J Agric Food Chem. 2002; 50(16): 4437-44

[12] GenStat, Eleventh, Edition. Hemel Hempstead, UK: VSN International Ltd.; 2008.

[13] Famiani F, Cultrera NGM, Battistelli A, Casulli V, Proietti P, Standardi A, et al. Phosphoenolpyruvate carboxykinase and its potential role in the catabolism of organic acids in the flesh of soft fruit during ripening. J Exp Bot. 2005; 56(421): $2959-69$.

[14] Kader F, Rovel B, Metche M. Role of Invertase in Sugar Content in Highbush Blueberries (Vaccinium corymbosum, L.). LWT - Food Science and Technology. 1993; 26(6): 593-5.

[15] Kushman LJ, Ballinger WE. Acid and sugar changes during ripening in Wolcott blueberries. Proceedings of the American Society for Horticultural Science. 1968; 92: 290-5.

[16] Markakis P, Jarczyk A, Krishna SP. Nonvolatile acids of blueberries. J Agric Food Chem. 1963; 11: 8-11.

[17] Brady CJ. Fruit ripening. Annual Review of Plant Physiology. [Surveys.] 1987; 38(38): 155-78.

[18] Hong V, Wrolstad RE. Cranberry juice composition. Journal of the Association of Official Analytical Chemists. 1986; 69(2): $199-207$.

[19] Özgen M, Palta JP, Smith JD. Ripeness stage at harvest influences postharvest life of cranberry fruit: Physiological and anatomical explanations. Postharvest Biol Technol. 2002; 24(3): 291-9.

[20] Kalt W, MacKinnon S, McDonald J, Vinqvist M, Craft C, Howell A. Phenolics of Vaccinium berries and other fruit crops. J Sci Food Agric. 2008; 88(1): 68-76. 